\title{
Blueberry Powder Supplement
}

National Cancer Institute

\section{Source}

National Cancer Institute. Blueberry Powder Supplement. NCI Thesaurus. Code C98108.

An orally available, dietary supplement consisting of lyophilized blueberry powder, with antioxidant and potential chemopreventive and chemosensitizing activity. In addition to vitamins and minerals, blueberries are rich in phytonutrients, such as proanthocyanidins, anthocyanins (e.g. malvidin, delphinidin, pelargonidin, cyanidin, petunidin, and peonidin), hydroxycinnamic acids, hydroxybenzoic acids, pterostilbene, resveratrol, and flavonols (e.g. kaempferol, quercetin and myricetin). Although the exact mechanism of action through which blueberries may exert their anti-tumor effect has yet to be fully elucidated, the effects of blueberry powder on cancer cells may be attributable to the phytonutrient's antioxidant and pro-apoptotic activities. 